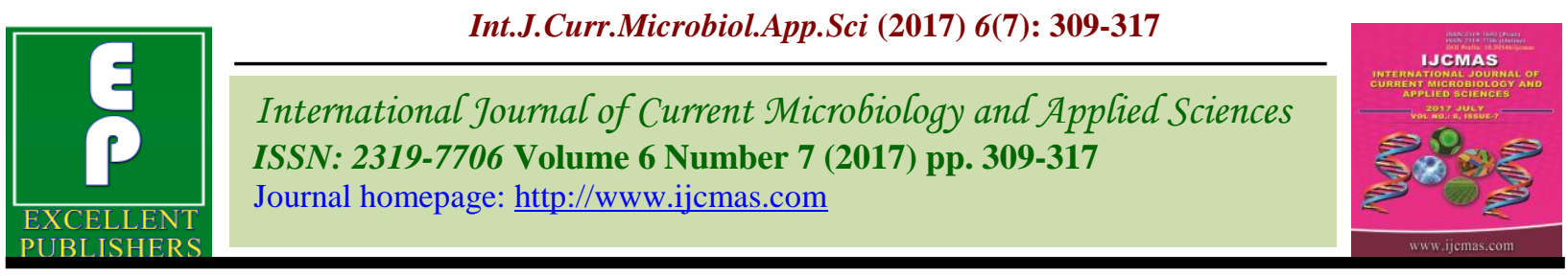

Original Research Article

https://doi.org/10.20546/ijcmas.2017.607.036

\title{
Bioefficacy of Cyantraniliprole 10\% OD W/V (HGW86 10 OD) against Pests of Potato
}

\author{
J.P. Lodaya, N.B. Patel", R.D. Patel and R.R. Acharya \\ Main Vegetable Research Station, Anand Agricultural University, \\ Anand-388110 (Gujarat), India \\ *Corresponding author
}

\section{A B S T R A C T}

\begin{tabular}{|l|}
\hline K e y w or d s \\
Cyantraniliprole \\
$10 \%$ OD, \\
Aphid, Thrips, \\
H. armigera, \\
Potato. \\
\hline Article Info \\
\hline $\begin{array}{l}\text { Accepted: } \\
\text { 04 June } 2017 \\
\text { Available Online: } \\
\text { 10 July } 2017\end{array}$ \\
\hline
\end{tabular}

A field experiment was conducted to evaluate the bio-efficacy of cyantraniliprole $10 \%$ OD against major insect pests infesting potato, Solanum tuberosum L. during rabi season of 2014 and 2015at Main Vegetable Research Station, Anand Agricultural University, Anand (Gujarat). During the course of study, three insect pest species, viz., aphid (Aphis gossypii Glover), thrips (Thrips tabaci Lindeman) and Helicoverpa armigera Hübner (Hardwick) were recorded on potato at various stages of the crop. The tested insecticide cyantraniliprole 10\% OD was evaluated at four doses i.e. 45, 60, 75 and $90 \mathrm{~g}$ a.i./ha along with dimethoate $30 \mathrm{EC}$ at $200 \mathrm{~g}$ a.i./ha and thiamethoxam $25 \mathrm{WG}$ at $25 \mathrm{~g}$ a.i./ha. The treatment of cyantraniliprole 10\% OD @60 g a.i./ha found effective as it provided excellent protection against aphid, thrips and $H$. armigera in potato. This treatment also manifested higher tuber yield and found safer to spider observed in the field.

\section{Introduction}

Potato (Solanum tuberosum L.) is the fourth most important food crop in the world after wheat, rice, and maize in terms of production. It contributes about $22 \%$ of the total vegetables and about $40 \%$ of the root and tuber crops produced in the world (FAO, 2001). India is the third largest producer of potato crop in the world and it is grown in a commercial scale (Khurana and Naik, 2003). The main constraint to potato farming in India is vulnerable to pests and diseases hence implying a high risk of failure, growing potatoes requires substantial capital and the crop needs intensive care and attention.Potatoes are reported to be attacked by more than 80 insects and many nematode pests in the field and in store in India (Misra and Agrawal, 2008). They can be broadly classified into soil pests, sucking pests and sap feeders, defoliators and storage pests viz., leaf eating caterpillar, serpentine leaf miner, Helicoverpa armigera, aphid, thrips, jassids and whitefly. Due to severe infestation of these pests, farmers fail to get higher yield even after frequent spray application of routine insecticides.Keeping in mind with these objectives, the bio-efficacy of cyantraniliprole was undertaken with recommended insecticides for the control of insect pests of potato. Scanty information is available of in efficacy on insect pests infesting potato. However, field bio-efficacy 
with different doses of cyantraniliprole was evaluated earlier by few workers (Patel et al., 2011; Mandal, 2012; Misra, 2012; Patel and Kher, 2012a and Patel and Kher, 2012b) on other crops.

Cyantraniliprole (IRAC MoA 28) is a second generation anthranilic diamide insecticide discovered by DuPont Crop Protection. It has unique mode of action targeting the ryanodinereceptors (RyR) in insect muscle cells (Sattelle et al., 2008; IRAC, 2012). Cyantraniliprole is the first to control a cross spectrum of chewing (Lepidoptera) and sucking (Hemiptera) pests (Anonymous, 2012). This group of insecticides also possesses the antifeedant properties (Gonzales-Coloma et al., 1999).

\section{Materials and Methods}

A field experiment was conducted during rabi season of 2014 and 2015 to assess efficacy of cyantraniliprole $10 \%$ OD against insect pests ofpotato in a Randomized Block Design (RBD) at Main Vegetable Research Station, Anand Agricultural University, Anand (Gujarat). For the purpose, potato variety Kufri Pukhraj was planted in a plot of $4.5 \mathrm{x}$ $3.0 \mathrm{~m}$ with a spacing of $45 \times 15 \mathrm{~cm}$. There were total four different doses of cyantraniliprole $10 \%$ OD tested and they are 45, 60, 75 and $90 \mathrm{~g}$ a.i./ha along with check treatments dimethoate $30 \mathrm{EC}$ at $200 \mathrm{~g}$ a.i./ha and thiamethoxam $25 \mathrm{WG}$ at $25 \mathrm{~g}$ a.i./ha. The respective treatments of insecticides were sprayed on potato crop by manually operated knapsack sprayer with hollow cone nozzle. Considering the activity of different pests, two sprays were given during the crop period.

For sucking pest count (aphid and thrips), five plants were randomly selected and tagged in each net plot and recorded during early morning hrs. Population of aphid and thrips were recorded from 3 leaves (upper, middle, lower) per plant prior and after 3, 7 and 10 days of each spray. The data obtained were analyzed periodically, spray-wise as well as pooled over periods and sprays. The larval population of $H$. armigera, five plants were randomly selected and tagged in each net plot. Population of $H$. armigera larvae were recorded from the selected plants prior and after 3, 7 and 10 days of each spray. The data obtained were analyzed periodically, spraywise as well as pooled over periods and sprays. In case of natural enemies, population was recorded before and after 3, 7 and 10 days of each spray from five randomly selected plants. The data of insect population were subjected to square root transformation for analysis.

\section{Results and Discussion}

\section{Aphid}

The data on pooled over periods and sprays on number of aphids per three leaves during first year are presented in Table 1. The data revealed among the different doses of cyantraniliprole $10 \% \mathrm{OD}$, the highest dose i.e. $90 \mathrm{~g}$ a.i./ha registered the lowest (1.16/ 3 leaves) aphid population than rest of the treatments, however, it remained at par with later two doses 75 and $60 \mathrm{~g}$ a.i./ha, which harboured the pest population of 1.24 and 1.32 per 3 leaves, respectively. The lowest dose (45 g a.i./ha) of cyantraniliprole $10 \%$ OD (2.74/ 3 leaves) was statistically inferior to its higher rates and remained at par with thiamethoxam $25 \mathrm{WG}(2.67 / 3$ leaves $)$ but superior to dimethoate $30 \mathrm{EC}$ (4.70/ 3 leaves).

During second year, the data on pooled over periods and sprays showed significant effectiveness of all the insecticidal treatments when compared with control. The highest dose $90 \mathrm{~g}$ a.i./ha of cyantraniliprole $10 \%$ OD found significantly effective in reducing aphid 
population ( 0.78 aphids/ 3 leaves) than rest of the treatments except 60 and $75 \mathrm{~g}$ a.i./ha of cyantraniliprole $10 \%$ OD with 0.80 and 0.82 aphids per 3 leaves, respectively and it was followed by thiamethoxam $25 \mathrm{WG}$ (2.26 aphids/ 3 leaves) and the lowest dose of cyantraniliprole 10\% OD @ 45 g a.i./ha (2.29 aphids/ 3 leaves).

Based on pooled results of both the years on bio-efficacy of cyantraniliprole $10 \%$ OD against aphid indicated that the plots treated with cyantraniliprole 10\% OD @ 60,75 and $90 \mathrm{~g}$ a.i./ha were significantly effective in controlling aphids in potato over rest of the treatments and these three treatments were at par with each other. The superiority of cyantraniliprole against $A$. gossypii revealed in present study is in accordance with the report of Mandal (2012) who reported that cyantraniliprole @ 90 and 105 g a.i./ha was more effective in reducing the pest population in tomato.

\section{Thrips}

The pooled result of two sprays during first year on thrips population (Table 2) recorded in different treatments indicated that the plots treated with cyantraniliprole $10 \%$ OD provided superior protection against thrips compared to standard checks and untreated control. Among the various doses of cyantraniliprole $10 \%$ OD, the higher three doses (90, 75 and $60 \mathrm{~g}$ a.i./ha) depicted the pest population between 0.48 and 0.69 thrips per three leaves and observed as significantly superior to its lower dose of $45 \mathrm{~g}$ a.i./ha, thiamethoxam $25 \mathrm{WG}$ and dimethoate $30 \mathrm{EC}$ $(1.43,1.60$ and 2.81 per three leaves, respectively).

The result of two sprays on thrips population during second year illustrated that plots treated with cyantraniliprole $10 \%$ OD showed its superiority in suppressing thrips population over rest of the treatments. Plots treated with the highest dose of cyantraniliprole 10\% OD @ $90 \mathrm{~g}$ a.i./ha recorded the lowest (0.69 thrips/ 3 leaves) and it was at par with its subsequent two doses i.e. 60 and $75 \mathrm{~g}$ a.i./ha (0.85 thrips/ 3 leaves). While, the remaining treatments of thiamethoxam $25 \mathrm{WG}$ and cyantraniliprole $10 \%$ OD @ 45 g a.i./ha remained at par with each other and recorded higher (1.63 and 1.81) number of thrips population per three leaves than former treatments.

The pooled data of two years on bio-efficacy of cyantraniliprole 10\% OD against thrips indicated that the plots treated with cyantraniliprole 10\% OD @ 60, 75 and 90 g a.i./ha were significantly effective in controlling thrips in potato over rest of the treatments and these three treatments were at par with each other. On the other hand, treatment of dimethoate $30 \mathrm{EC}$ (3.03 thrips/ 3 leaves) proved least effective with higher number of thrips than the lowest dose of cyantraniliprole 10\% OD @ $45 \mathrm{~g}$ a.i./ha and thiamethoxam 25 WG (1.60 thrips/ 3 leaves).

Patel et al., (2014) reported that two higher doses of cyantraniliprole 10\% OD i.e. 90 and $105 \mathrm{~g}$ a.i./ha wasfound highly effective in managing the population of aphid, thrips and whitefly in cotton. According to Misra (2012), both the doses of cyantraniliprole i.e. 105 and $90 \mathrm{~g}$ a.i./ha were found equally effective against $T$. tabaci infesting tomato. This is in agreement with the present finding.

\section{H. armigera}

The larval population of $H$. armigera (Table 3 ) was significantly lower in all treated plots over control as it evident from the observations recorded during $1^{\text {st }}$ year. The data on pooled over periods and sprays showed that the plots treated with cyantraniliprole $10 \%$ OD @ 90 g a.i./ha 
proved effective and recorded the lowest (0.67 larvae/ plant) number of larvae than remaining treatments. However, treatment of cyantraniliprole 10\% OD @ 75 and $60 \mathrm{~g}$ a.i./ha registered lower ( 0.78 and 0.82 larvae/ plant) number of larvae and remained at par with the highest dose of cyantraniliprole $10 \%$
OD and it was followed by the lowest dose of cyantraniliprole 10\% OD @ $45 \mathrm{~g}$ a.i./ha (1.87 larvae/ plant). Whereas, treatment of dimethoate $30 \mathrm{EC}$ (3.54 larvae/ plant) and thiamethoxam 25 WG (3.62 larvae/ plant) remained at par with each other and proved less effective in suppressing the pest.

Table.1 Effect of different insecticides against aphid in potato (Pooled over years)

\begin{tabular}{|c|c|c|c|c|c|c|}
\hline \multirow{2}{*}{ Treatments (g a.i./ha) } & \multicolumn{6}{|c|}{ Number of aphid/ 3 leaves } \\
\hline & \multicolumn{2}{|c|}{2014} & \multicolumn{2}{|c|}{2015} & \multicolumn{2}{|c|}{ Pooled } \\
\hline Cyantraniliprole 10 OD (45) & \multicolumn{2}{|c|}{$\begin{array}{l}1.80 b^{*} \\
(2.74)\end{array}$} & \multicolumn{2}{|c|}{$\begin{array}{l}1.67 b \\
(2.29)\end{array}$} & \multicolumn{2}{|c|}{$\begin{array}{l}1.73 b \\
(2.49)\end{array}$} \\
\hline Cyantraniliprole 10 OD (60) & \multicolumn{2}{|c|}{$\begin{array}{l}1.35 \mathrm{a} \\
(1.32)\end{array}$} & \multicolumn{2}{|c|}{$\begin{array}{l}1.14 \mathrm{a} \\
(0.80)\end{array}$} & \multicolumn{2}{|c|}{$\begin{array}{l}1.24 \mathrm{a} \\
(1.04) \\
\end{array}$} \\
\hline Cyantraniliprole 10 OD (75) & \multicolumn{2}{|c|}{$\begin{array}{l}1.32 \mathrm{a} \\
(1.24)\end{array}$} & \multicolumn{2}{|c|}{$\begin{array}{l}1.15 \mathrm{a} \\
(0.82)\end{array}$} & \multicolumn{2}{|c|}{$\begin{array}{l}1.24 \mathrm{a} \\
(1.04)\end{array}$} \\
\hline Cyantraniliprole 10 OD (90) & \multicolumn{2}{|c|}{$\begin{array}{l}1.29 \mathrm{a} \\
(1.16)\end{array}$} & \multicolumn{2}{|c|}{$\begin{array}{c}1.13 \mathrm{a} \\
(0.78)\end{array}$} & \multicolumn{2}{|c|}{$\begin{array}{r}1.21 \mathrm{a} \\
(0.96) \\
\end{array}$} \\
\hline Thiamethoxam 25 WG (25) & \multicolumn{2}{|c|}{$\begin{array}{l}1.78 b \\
(2.67)\end{array}$} & \multicolumn{2}{|c|}{$\begin{array}{l}1.66 b \\
(2.26)\end{array}$} & \multicolumn{2}{|c|}{$\begin{array}{l}1.72 b \\
(2.46)\end{array}$} \\
\hline Dimethoate 30 EC (200) & \multicolumn{2}{|c|}{$\begin{array}{l}2.28 \mathrm{c} \\
(4.70)\end{array}$} & \multicolumn{2}{|c|}{$\begin{array}{l}2.16 c \\
(4.17)\end{array}$} & \multicolumn{2}{|c|}{$\begin{array}{l}2.22 \mathrm{c} \\
(4.43)\end{array}$} \\
\hline \multirow[t]{2}{*}{ Untreated Control } & \multicolumn{2}{|c|}{$\begin{array}{l}2.79 d \\
(7.28)\end{array}$} & \multicolumn{2}{|c|}{$\begin{array}{l}2.74 d \\
(7.01)\end{array}$} & \multicolumn{2}{|c|}{$\begin{array}{l}2.76 \mathrm{~d} \\
(7.12)\end{array}$} \\
\hline & $\begin{array}{c}\text { S. Em. } \\
\pm\end{array}$ & $\begin{array}{c}\text { CD } \\
(5 \%)\end{array}$ & S. Em. \pm & $\mathrm{CD}(5 \%)$ & $\begin{array}{c}\text { S. } \\
\text { Em. } \pm\end{array}$ & $\begin{array}{c}\text { CD } \\
(5 \%)\end{array}$ \\
\hline Treatment $(\mathbf{T})$ & 0.04 & 0.12 & 0.04 & 0.12 & 0.03 & 0.08 \\
\hline Period (P) & 0.03 & 0.08 & 0.03 & 0.07 & 0.08 & NS \\
\hline Spray (S) & 0.02 & 0.06 & 0.02 & NS & 0.02 & 0.04 \\
\hline Year $(Y)$ & - & - & - & - & 0.02 & 0.04 \\
\hline $\mathbf{T} \times \mathbf{P}$ & 0.06 & 0.16 & 0.06 & NS & 0.05 & NS \\
\hline $\mathbf{T} \times S$ & 0.04 & NS & 0.04 & NS & 0.04 & NS \\
\hline$T \times Y$ & - & - & - & - & 0.04 & NS \\
\hline $\mathbf{P} \times S$ & 0.07 & NS & 0.07 & 0.20 & 0.06 & NS \\
\hline $\mathbf{P} \times \mathbf{Y}$ & - & NS & - & - & 0.03 & NS \\
\hline$S \times Y$ & - & - & - & - & 0.03 & NS \\
\hline$T \times P \times S$ & 0.10 & NS & 0.10 & NS & 0.07 & 0.19 \\
\hline$T \times P \times Y$ & - & - & - & - & 0.07 & NS \\
\hline$T \times S \times Y$ & - & - & - & - & 0.06 & NS \\
\hline$P \times S \times Y$ & - & - & - & - & 0.04 & 0.10 \\
\hline$T \times P \times S \times Y$ & - & - & - & - & 0.10 & NS \\
\hline C. V. \% & \multicolumn{2}{|c|}{9.60} & \multicolumn{2}{|c|}{10.19} & \multicolumn{2}{|c|}{9.82} \\
\hline
\end{tabular}

* Figures in parentheses are retransformed values; those outside are $\sqrt{X+0.5}$ transformed values; NS $=$ Not significant 
Table.2 Effect of different insecticides against thrips in potato (Pooled over years)

\begin{tabular}{|c|c|c|c|c|c|c|}
\hline \multirow{2}{*}{ Treatments (g a.i./ha) } & \multicolumn{6}{|c|}{ Number of thrips/ 3 leaves } \\
\hline & \multicolumn{2}{|c|}{2014} & \multicolumn{2}{|c|}{2015} & \multicolumn{2}{|c|}{ Pooled } \\
\hline $\begin{array}{l}\text { Cyantraniliprole } 10 \\
\text { OD (45) }\end{array}$ & \multicolumn{2}{|c|}{$\begin{array}{l}1.39 b^{*} \\
(1.43) \\
\end{array}$} & \multicolumn{2}{|c|}{$\begin{array}{l}1.52 \mathrm{~b} \\
(1.81)\end{array}$} & \multicolumn{2}{|c|}{$\begin{array}{l}1.45 \mathrm{~b} \\
(1.60)\end{array}$} \\
\hline $\begin{array}{l}\text { Cyantraniliprole } 10 \\
\text { OD (60) }\end{array}$ & \multicolumn{2}{|c|}{$\begin{array}{l}1.09 \mathrm{a} \\
(0.69)\end{array}$} & \multicolumn{2}{|c|}{$\begin{array}{l}1.16 a \\
(0.85)\end{array}$} & \multicolumn{2}{|c|}{$\begin{array}{l}1.13 a \\
(0.78)\end{array}$} \\
\hline $\begin{array}{l}\text { Cyantraniliprole } 10 \\
\text { OD (75) }\end{array}$ & \multicolumn{2}{|c|}{$\begin{array}{l}1.06 \mathrm{a} \\
(0.62)\end{array}$} & \multicolumn{2}{|c|}{$\begin{array}{l}1.16 \mathrm{a} \\
(0.85)\end{array}$} & \multicolumn{2}{|c|}{$\begin{array}{l}1.11 \mathrm{a} \\
(0.73)\end{array}$} \\
\hline $\begin{array}{l}\text { Cyantraniliprole } 10 \\
\text { OD (90) }\end{array}$ & \multicolumn{2}{|c|}{$\begin{array}{l}0.99 \mathrm{a} \\
(0.48)\end{array}$} & \multicolumn{2}{|c|}{$\begin{array}{l}1.09 \mathrm{a} \\
(0.69)\end{array}$} & \multicolumn{2}{|c|}{$\begin{array}{l}1.04 \mathrm{a} \\
(0.58)\end{array}$} \\
\hline $\begin{array}{l}\text { Thiamethoxam } 25 \text { WG } \\
\text { (25) }\end{array}$ & \multicolumn{2}{|c|}{$\begin{array}{l}1.45 \mathrm{~b} \\
(1.60)\end{array}$} & \multicolumn{2}{|c|}{$\begin{array}{l}1.46 \mathrm{~b} \\
(1.63)\end{array}$} & \multicolumn{2}{|c|}{$\begin{array}{l}1.45 \mathrm{~b} \\
(1.60)\end{array}$} \\
\hline $\begin{array}{l}\text { Dimethoate } 30 \text { EC } \\
\text { (200) }\end{array}$ & \multicolumn{2}{|c|}{$\begin{array}{l}1.82 \mathrm{c} \\
(2.81)\end{array}$} & \multicolumn{2}{|c|}{$\begin{array}{l}1.95 \mathrm{c} \\
(3.30)\end{array}$} & \multicolumn{2}{|c|}{$\begin{array}{l}1.88 \mathrm{c} \\
(3.03)\end{array}$} \\
\hline Untreated Control & \multicolumn{2}{|c|}{$\begin{array}{l}2.36 \mathrm{~d} \\
(5.07)\end{array}$} & \multicolumn{2}{|c|}{$\begin{array}{l}2.90 \mathrm{~d} \\
(7.91)\end{array}$} & \multicolumn{2}{|c|}{$\begin{array}{l}2.63 d \\
(6.42)\end{array}$} \\
\hline & S. Em. \pm & $\mathrm{CD}(5 \%)$ & S. Em. \pm & $\mathrm{CD}(5 \%)$ & S. Em. \pm & $\mathrm{CD}(5 \%)$ \\
\hline Treatment $(\mathrm{T})$ & 0.04 & 0.13 & 0.04 & 0.11 & 0.09 & 0.30 \\
\hline Period (P) & 0.03 & 0.07 & 0.02 & 0.07 & 0.04 & NS \\
\hline Spray (S) & 0.02 & NS & 0.02 & NS & 0.002 & NS \\
\hline Year $(\mathbf{Y})$ & - & - & - & - & 0.02 & 0.04 \\
\hline $\mathbf{T} \times \mathbf{P}$ & 0.06 & 0.16 & 0.05 & NS & 0.05 & 0.13 \\
\hline $\mathbf{T} \times \mathbf{S}$ & 0.04 & 0.10 & 0.03 & NS & 0.04 & 0.10 \\
\hline $\mathbf{T} \times \mathbf{Y}$ & - & - & - & - & 0.04 & 0.11 \\
\hline $\mathbf{P} \times \mathbf{S}$ & 0.07 & 0.19 & 0.06 & 0.17 & 0.07 & NS \\
\hline $\mathbf{P} \times \mathbf{Y}$ & - & - & - & - & 0.02 & 0.07 \\
\hline $\mathbf{S x Y}$ & - & - & - & - & 0.02 & 0.07 \\
\hline$T \times P \times S$ & 0.10 & 0.27 & 0.09 & 0.25 & 0.07 & 0.18 \\
\hline$T \times P \times Y$ & - & - & - & - & 0.07 & NS \\
\hline $\mathbf{T} \times S \times Y$ & - & - & - & - & 0.05 & NS \\
\hline$P \times S \times Y$ & - & - & - & - & 0.04 & 0.10 \\
\hline$T \times P \times S \times Y$ & - & - & - & - & 0.09 & NS \\
\hline C. V. \% & \multicolumn{2}{|c|}{11.37} & \multicolumn{2}{|c|}{9.43} & \multicolumn{2}{|c|}{10.38} \\
\hline
\end{tabular}

* Figures in parentheses are retransformed values; those outside are $\sqrt{X+0.5}$ transformed values; NS = Not significant 
Table.3 Effect of different insecticides against larval population of H. armigera in potato (Pooled over years)

\begin{tabular}{|c|c|c|c|c|c|c|}
\hline \multirow{2}{*}{$\begin{array}{l}\text { Treatments (g } \\
\text { a.i./ha) }\end{array}$} & \multicolumn{6}{|c|}{ Number of larvae/ plant } \\
\hline & \multicolumn{2}{|c|}{2014} & \multicolumn{2}{|c|}{2015} & \multicolumn{2}{|c|}{ Pooled } \\
\hline $\begin{array}{l}\text { Cyantraniliprole } 10 \\
\text { OD (45) }\end{array}$ & \multicolumn{2}{|c|}{$\begin{array}{l}1.54 b^{*} \\
(1.87)\end{array}$} & \multicolumn{2}{|c|}{$\begin{array}{l}1.39 \mathrm{~b} \\
(1.43)\end{array}$} & \multicolumn{2}{|c|}{$\begin{array}{l}1.47 \mathrm{~b} \\
(1.66)\end{array}$} \\
\hline $\begin{array}{l}\text { Cyantraniliprole } 10 \\
\text { OD }(60)\end{array}$ & \multicolumn{2}{|c|}{$\begin{array}{l}1.15 \mathrm{a} \\
(0.82)\end{array}$} & \multicolumn{2}{|c|}{$\begin{array}{l}1.07 \mathrm{a} \\
(0.64)\end{array}$} & \multicolumn{2}{|c|}{$\begin{array}{l}1.11 \mathrm{a} \\
(0.73)\end{array}$} \\
\hline $\begin{array}{l}\text { Cyantraniliprole } 10 \\
\text { OD (75) }\end{array}$ & \multicolumn{2}{|c|}{$\begin{array}{l}1.13 a \\
(0.78)\end{array}$} & \multicolumn{2}{|c|}{$\begin{array}{c}1.05 a \\
(0.60)\end{array}$} & \multicolumn{2}{|c|}{$\begin{array}{c}1.09 \mathrm{a} \\
(0.69)\end{array}$} \\
\hline $\begin{array}{l}\text { Cyantraniliprole } 10 \\
\text { OD (90) }\end{array}$ & \multicolumn{2}{|c|}{$\begin{array}{l}1.08 \mathrm{a} \\
(0.67)\end{array}$} & \multicolumn{2}{|c|}{$\begin{array}{l}1.00 \mathrm{a} \\
(0.51)\end{array}$} & \multicolumn{2}{|c|}{$\begin{array}{l}1.04 \mathrm{a} \\
(0.58)\end{array}$} \\
\hline $\begin{array}{l}\text { Thiamethoxam } 25 \\
\text { WG (25) }\end{array}$ & \multicolumn{2}{|c|}{$\begin{array}{l}2.03 \mathrm{c} \\
(3.62)\end{array}$} & \multicolumn{2}{|c|}{$\begin{array}{l}1.84 \mathrm{c} \\
(2.89)\end{array}$} & \multicolumn{2}{|c|}{$\begin{array}{l}1.93 \mathrm{c} \\
(3.22)\end{array}$} \\
\hline $\begin{array}{l}\text { Dimethoate } 30 \text { EC } \\
\text { (200) }\end{array}$ & \multicolumn{2}{|c|}{$\begin{array}{l}2.01 \mathrm{c} \\
(3.54)\end{array}$} & \multicolumn{2}{|c|}{$\begin{array}{l}1.92 \mathrm{c} \\
(3.19)\end{array}$} & \multicolumn{2}{|c|}{$\begin{array}{l}1.97 \mathrm{c} \\
(3.38)\end{array}$} \\
\hline \multirow[t]{2}{*}{ Untreated Control } & \multicolumn{2}{|c|}{$\begin{array}{l}2.41 \mathrm{~d} \\
(5.31)\end{array}$} & \multicolumn{2}{|c|}{$\begin{array}{l}2.12 \mathrm{~d} \\
(3.99)\end{array}$} & \multicolumn{2}{|c|}{$\begin{array}{l}2.27 d \\
(4.65)\end{array}$} \\
\hline & S. Em. \pm & $\mathrm{CD}(5 \%)$ & S. Em. \pm & $\mathrm{CD}(5 \%)$ & S. Em. \pm & $\mathrm{CD}(5 \%)$ \\
\hline Treatment $(\mathbf{T})$ & 0.04 & 0.13 & 0.03 & 0.08 & 0.04 & 0.14 \\
\hline Period $(\mathbf{P})$ & 0.02 & NS & 0.02 & NS & 0.04 & 0.13 \\
\hline Spray (S) & 0.02 & NS & 0.02 & 0.06 & 0.01 & NS \\
\hline Year $(\mathbf{Y})$ & - & - & - & - & 0.01 & 0.04 \\
\hline $\mathbf{T} \times \mathbf{P}$ & 0.05 & NS & 0.05 & NS & 0.05 & 0.13 \\
\hline $\mathbf{T} \times \mathbf{S}$ & 0.03 & NS & 0.03 & NS & 0.04 & NS \\
\hline $\mathbf{T} \times \mathbf{Y}$ & - & - & - & - & 0.04 & 0.10 \\
\hline $\mathbf{P} \times \mathbf{S}$ & 0.06 & NS & 0.07 & NS & 0.02 & NS \\
\hline $\mathbf{P} \times \mathbf{Y}$ & - & - & - & - & 0.02 & NS \\
\hline $\mathbf{S} \times \mathbf{Y}$ & - & - & - & - & 0.03 & NS \\
\hline $\mathbf{T} \times \mathbf{P} \times \mathrm{S}$ & 0.09 & NS & 0.09 & NS & 0.06 & NS \\
\hline $\mathbf{T} \times \mathbf{P} \times \mathbf{Y}$ & - & - & - & - & 0.06 & NS \\
\hline $\mathbf{T} \times S \times Y$ & - & - & - & - & 0.06 & NS \\
\hline$P \times S \times Y$ & - & - & - & - & 0.03 & NS \\
\hline $\mathbf{T} \times \mathbf{P} \times S \times Y$ & - & - & - & - & 0.09 & NS \\
\hline C. V. \% & \multicolumn{2}{|c|}{9.40} & \multicolumn{2}{|c|}{10.80} & \multicolumn{2}{|c|}{9.99} \\
\hline
\end{tabular}

* Figures in parentheses are retransformed values; those outside are $\sqrt{X+0.5}$ transformed values; NS = Not significant 
Table.4 Impact of different insecticides on spiders in potato (Pooled over years)

\begin{tabular}{|c|c|c|c|c|c|c|}
\hline \multirow{2}{*}{ Treatments (g a.i./ha) } & \multicolumn{6}{|c|}{ Number of spiders/ plant } \\
\hline & \multicolumn{2}{|c|}{2014} & \multicolumn{2}{|c|}{2015} & \multicolumn{2}{|c|}{ Pooled } \\
\hline $\begin{array}{l}\text { Cyantraniliprole 10 OD } \\
\text { (45) }\end{array}$ & \multicolumn{2}{|c|}{$\begin{array}{l}1.07 * \\
(0.64)\end{array}$} & \multicolumn{2}{|c|}{$\begin{array}{c}1.15 \\
(0.82)\end{array}$} & \multicolumn{2}{|c|}{$\begin{array}{c}1.11 \\
(0.73)\end{array}$} \\
\hline $\begin{array}{l}\text { Cyantraniliprole } 10 \text { OD } \\
\text { (60) }\end{array}$ & \multicolumn{2}{|c|}{$\begin{array}{c}1.07 \\
(0.64)\end{array}$} & \multicolumn{2}{|c|}{$\begin{array}{c}1.18 \\
(0.89)\end{array}$} & \multicolumn{2}{|c|}{$\begin{array}{c}1.12 \\
(0.75)\end{array}$} \\
\hline $\begin{array}{l}\text { Cyantraniliprole 10 OD } \\
\text { (75) }\end{array}$ & \multicolumn{2}{|c|}{$\begin{array}{c}1.09 \\
(0.69)\end{array}$} & \multicolumn{2}{|c|}{$\begin{array}{c}1.13 \\
(0.78)\end{array}$} & \multicolumn{2}{|c|}{$\begin{array}{c}1.11 \\
(0.73)\end{array}$} \\
\hline $\begin{array}{l}\text { Cyantraniliprole } 10 \text { OD } \\
\text { (90) }\end{array}$ & \multicolumn{2}{|c|}{$\begin{array}{l}1.01 \\
(0.52)\end{array}$} & \multicolumn{2}{|c|}{$\begin{array}{c}1.08 \\
(0.67)\end{array}$} & \multicolumn{2}{|c|}{$\begin{array}{c}1.05 \\
(0.60)\end{array}$} \\
\hline $\begin{array}{l}\text { Thiamethoxam } 25 \text { WG } \\
\text { (25) }\end{array}$ & \multicolumn{2}{|c|}{$\begin{array}{c}1.00 \\
(0.50)\end{array}$} & \multicolumn{2}{|c|}{$\begin{array}{c}1.11 \\
(0.73)\end{array}$} & \multicolumn{2}{|c|}{$\begin{array}{c}1.06 \\
(0.62)\end{array}$} \\
\hline Dimethoate 30 EC (200) & \multicolumn{2}{|c|}{$\begin{array}{c}1.02 \\
(0.54)\end{array}$} & \multicolumn{2}{|c|}{$\begin{array}{c}1.10 \\
(0.71)\end{array}$} & \multicolumn{2}{|c|}{$\begin{array}{c}1.06 \\
(0.62)\end{array}$} \\
\hline Untreated Control & \multicolumn{2}{|c|}{$\begin{array}{c}1.09 \\
(1.69)\end{array}$} & \multicolumn{2}{|c|}{$\begin{array}{c}1.20 \\
(0.94)\end{array}$} & \multicolumn{2}{|c|}{$\begin{array}{c}1.15 \\
(0.82)\end{array}$} \\
\hline & S. Em. \pm & CD $(5 \%)$ & S. Em. \pm & CD (5\%) & S. Em. \pm & CD (5\%) \\
\hline Treatment $(\mathbf{T})$ & 0.05 & NS & 0.03 & NS & 0.03 & NS \\
\hline Period (P) & 0.01 & NS & 0.02 & NS & 0.01 & 0.03 \\
\hline Spray (S) & 0.01 & NS & 0.02 & NS & 0.01 & NS \\
\hline Year $(\mathbf{Y})$ & - & - & - & - & 0.01 & 0.04 \\
\hline $\mathbf{T} \times \mathbf{P}$ & 0.03 & NS & 0.05 & NS & 0.03 & NS \\
\hline$T \times S$ & 0.02 & NS & 0.03 & NS & 0.03 & NS \\
\hline$T \times Y$ & - & - & - & - & 0.04 & NS \\
\hline $\mathbf{P} \times \mathrm{S}$ & 0.03 & NS & 0.06 & NS & 0.04 & NS \\
\hline $\mathbf{P} \times \mathbf{Y}$ & - & - & - & - & 0.02 & NS \\
\hline $\mathbf{S x Y}$ & - & - & - & - & 0.02 & NS \\
\hline$T \times P \times S$ & 0.05 & NS & 0.08 & NS & 0.05 & NS \\
\hline $\mathbf{T} \times \mathbf{P} \times \mathbf{Y}$ & - & - & - & - & 0.05 & NS \\
\hline$T \times S \times Y$ & - & - & - & - & 0.04 & NS \\
\hline$P \times S \times Y$ & - & - & - & - & 0.02 & NS \\
\hline$T \times P \times S \times Y$ & - & - & - & - & 0.07 & NS \\
\hline C. V. \% & \multicolumn{2}{|c|}{7.85} & \multicolumn{2}{|c|}{11.91} & \multicolumn{2}{|c|}{10.25} \\
\hline
\end{tabular}

* Figures in parentheses are retransformed values; those outside are $\sqrt{X+0.5}$ transformed values; NS = Not significant

Table.5 Effect of different insecticides on yield of potato (Pooled over years)

\begin{tabular}{|c|c|c|c|}
\hline \multirow{2}{*}{ Treatments (g a.i./ha) } & \multicolumn{3}{|c|}{ Yield (t/ha) } \\
\hline & 2014 & 2015 & Pooled \\
\hline Cyantraniliprole 10 OD (45) & $17.95 \mathrm{~b}$ & $19.07 \mathrm{~b}$ & $18.51 \mathrm{~b}$ \\
\hline Cyantraniliprole 10 OD (60) & $24.80 \mathrm{a}$ & $24.38 \mathrm{a}$ & $24.59 \mathrm{a}$ \\
\hline Cyantraniliprole 10 OD (75) & $25.08 \mathrm{a}$ & $24.83 \mathrm{a}$ & $24.96 \mathrm{a}$ \\
\hline Cyantraniliprole 10 OD (90) & $25.58 \mathrm{a}$ & $26.25 \mathrm{a}$ & $25.92 \mathrm{a}$ \\
\hline Thiamethoxam 25 WG (25) & $18.10 \mathrm{~b}$ & $19.03 \mathrm{~b}$ & $18.57 \mathrm{~b}$ \\
\hline Dimethoate 30 EC (200) & $12.60 \mathrm{c}$ & $13.93 \mathrm{c}$ & $13.27 \mathrm{c}$ \\
\hline Untreated Control & $5.53 \mathrm{~d}$ & $7.42 \mathrm{~d}$ & $6.48 \mathrm{~d}$ \\
\hline S. Em. \pm T & 1.18 & 1.18 & 0.77 \\
\hline $\mathbf{Y}$ & - & - & 0.45 \\
\hline TXY & - & - & 1.18 \\
\hline C. D. at 5\% T & 3.64 & 3.62 & 2.22 \\
\hline $\mathbf{Y}$ & - & - & 1.38 \\
\hline TXY & - & - & NS \\
\hline C.V. (\%) & 11.06 & 10.57 & 10.81 \\
\hline
\end{tabular}


The data on pooled over periods and sprays of second year indicated that among the different doses of cyantraniliprole $10 \%$ OD evaluated, the plots treated with $90 \mathrm{~g}$ a.i./ha registered the least (0.51 larvae/ plant) population of larvae. However, treatment of cyantraniliprole $10 \%$ OD @ 75 and $60 \mathrm{~g}$ a.i./ha (0.60 and 0.64 larvae/ plant) remained at par with the highest dose, but found statistically superior to the lowest dose of cyantraniliprole 10\% OD @ $45 \mathrm{~g}$ a.i./ha (1.43 larvae/ plant).

The data on pooled over years indicated that treatment of cyantraniliprole 10\% OD @ $90 \mathrm{~g}$ a.i./ha registered significantly the least $(0.58$ larvae/ plant) number of larvae than the rest of treatments except cyantraniliprole 10\% OD @ 75 and $60 \mathrm{~g}$ a.i./ha (0.69 and 0.73 larvae/ plant) with which it remained at par. However, treatment of cyantraniliprole 10\% OD @ $45 \mathrm{~g}$ a.i./ha recorded significantly lower (1.66 larvae/ plant) number of larvae than thiamethoxam 25 WG (3.22 larvae/ plant), dimethoate $30 \mathrm{EC}$ (3.38 larvae/ plant) and untreated control (4.65 larvae/ plant).The results are in agreement with the findings of Mandal (2012) reported the higher effectiveness of cyantraniliprole@90 and $105 \mathrm{~g}$ a.i./ha against the fruit borer, H. armigera in tomato.

\section{Natural Enemies}

The population of natural enemies prevailing in potato ecosystem was very low. Population of Chrysoperla and coccinellids was not found in potato crop even in the control plots, but population of spiders was observed during both the seasons (Table 4).There was no significant impact of any of the insecticidal treatments after its application as the result was non-significant. Data clearly revealed that all the insecticidal treatments including all the four different doses of cyantraniliprole $10 \%$ OD found more or less equally safer to the population of spiders. Shah et al., (2012) reported the safety of anthranilic diamide group of insecticide chlorantraniliprole to spider, which corroborates the present findings. In a nutshell, cyantraniliprole 10 OD was found safe to spider at all the tested doses and hence can easily be incorporated in an Integrated Pest Management programme.

\section{Yield}

The data on yield (Table 5) revealed that all the insecticidal treatments produced significantly higher potato tubers when compared with control during first and second year as well as in pooled. Among the different treatments, cyantraniliprole 10\% OD @ $90 \mathrm{~g}$ a.i./ha recorded significantly the highest yield of potato $(25.58 \mathrm{t} / \mathrm{ha})$ than the rest of treatments except cyantraniliprole 10\% OD @ 75 and $60 \mathrm{~g}$ a.i./ha (25.08 and $24.80 \mathrm{t} / \mathrm{ha})$ with which it remained at par during first year. The plots treated with thiamethoxam $25 \mathrm{WG}$ and the lowest dose of cyantraniliprole 10\% OD @ $45 \mathrm{~g}$ a.i./ha remained at par with each other and registered significantly higher (18.10 and $17.95 \mathrm{t} / \mathrm{ha})$ yield than dimethoate $30 \mathrm{EC}$ (12.60 t/ha) and untreated control (5.53 t/ha).

During second year, the data on yield of potato recorded in different treatments indicated that maximum (26.25 t/ha) yield was harvested from the plots sprayed with cyantraniliprole $10 \%$ OD @ $90 \mathrm{~g}$ a.i./ha and it was at par with treatments of cyantraniliprole 10\% OD @ 75 and $60 \mathrm{~g}$ a.i./ha (24.83 and $24.38 \mathrm{t} / \mathrm{ha}$ ). With respect to potato tuber yield, treatment of cyantraniliprole $10 \%$ OD @ $45 \mathrm{~g}$ a.i./ha and thiamethoxam $25 \mathrm{WG}$ remained at par with each other and recorded significantly higher yield than dimethoate $30 \mathrm{EC}$ $(13.93 \mathrm{t} / \mathrm{ha})$ as well as untreated control (7.42 tha).

Pooled data on yield of potato computed for two years revealed that the highest $(25.92 \mathrm{t} / \mathrm{ha})$ yield was harvested from the plots treated with

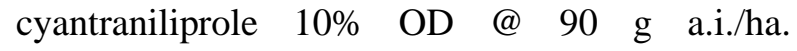
However, it remained at par with cyantraniliprole 10\% OD@ 75 and $60 \mathrm{~g}$ a.i./ha (24.96 and 24.59 t/ha). The plots treated with thiamethoxam $25 \mathrm{WG}$ and cyantraniliprole 10\% OD @ $45 \mathrm{~g}$ a.i./ha produced significantly higher (18.57 and 18.51 $\mathrm{t} / \mathrm{ha}$ ) yield over dimethoate $30 \mathrm{EC}(13.27 \mathrm{t} / \mathrm{ha})$ and untreated plot (6.48 t/ha).

It can be concluded among various evaluated doses of cyantraniliprole 10\% OD during two years of experimentation, cyantraniliprole 10\% OD @ $60 \mathrm{~g}$ a.i./ha found effective as it provided excellent protection against aphid, thrips and $H$. 
armigera in potato. This treatment also manifested higher tuber yield without any phytotoxic symptoms on the plant and safer to natural enemies observed in the field. Based on their efficacy levels as well as low toxicity to natural enemies, we conclude that cyantraniliprole $10 \%$ OD insecticides can be incorporated in future IPM programme in potato cultivation.

\section{Acknowledgement}

The authors thankful to M/S E. I. DuPont India Private Limited, Gurgaon, Haryana for the financial assistance given for testing of its newer product cyantraniliprole (Cyazypyr ${ }^{\mathrm{TM}} 10 \%$ OD).

\section{References}

Anonymous 2012. DuPont cyazypyr ${ }^{\mathrm{TM}}$ insect control. Tech. Bull. E. I., Du Pont de Nemours and Company. p. 4.

FAO 2001. Production Yearbook of Food and Agriculture Organization of the United Nations. Rome, Italy. 55: 97-98.

Gonzales-Coloma, A., Gutierrez, C., Hubner, H., Achenbach, H., Terrero, D. and Fraga, B. M. 1999. Selective insect anti-feedant and toxic action of ryanoid diterpenes. J. Agric. Food Chem. 47: 4419-4424.

IRAC 2012. IRAC Mode of Action Classification Scheme, Insecticide Resistance Action Committee, April, Version 7.2. pp. 1-23.

Khurana, S. M. P. and Naik, P. S. 2003. CPRI: Five decades of potato research and development. Souvenir of the National Symposium on Potato Research towards national Food and Nutritional Security, 2-3 October, 2003, CPRI, Shimla, pp.10.

Mandal, S. K. 2012. Bio-efficacy of cyazypyr $10 \%$ OD, a new anthranilic diamide insecticide against the insect pests of tomato and its impact on natural enemies and crop health. Acta Phytopathologica ET Entomologica Hungarica, 47(2): 233-249.

Misra, H. P. 2012. Field efficacy of a new molecule of insecticide against tomato thrips and its impact on coccinellid predators. SAARC J. Agri., 10(1): 63-70.

Misra, S. S. and Agrawal, Hari Om. 2008. Potato pests in India and their control. 34(2):199209.

Patel, J. J. and Kher, H. R. 2012a. Testing the bioefficacy and phytotoxicity of cyantraniliprole $10 \%$ OD against insect pests of okra. Final report, Main Vegetable Research Station, Anand Agricultural University, Anand. pp. 20-23.

Patel, J. J. and Kher, H. R. 2012b. Testing the bioefficacy and phytotoxicity of cyantraniliprole $10 \%$ OD against pests of brinjal. Final report, Main Vegetable Research Station, Anand Agricultural University, Anand. pp. 19-22.

Patel, J. J., Patel, H. C. and Kathiria, K. B. 2011. Testing the bio-efficacy and phytotoxicity of cyantraniliprole $10 \%$ OD against pests of tomato. Final report, Main Vegetable Research Station, Anand Agricultural University, Anand. pp. 16-18.

Patel, R. D., Bharpoda, T.M., Patel, N. B. and Borad, P. K. 2014. Bio-efficacy of cyantraniliprole 10\% OD - An Anthranilic Diamide insecticide against sucking pests of cotton. The Bioscan, 9(1): 89-92.

Sattelle, D. B., Cordova, D. and Cheek, T. R. 2008. Insect ryanodinereceptors: molecular targets for novel control chemicals. Invert. Neurosci. 8: 107-119.

Shah, K. D., Bharpoda, T. M. and Jhala, R. C. 2012. Bio-efficacy of newer molecules of insecticides against brinjal shoot and fruit borer, Leucinodes orbonalis Guenee (Lepidoptera: Pyralidae). AGRES- An International e-Journal, 1: 186-200.

\section{How to cite this article:}

Lodaya, J.P., N.B. Patel, R.D. Patel and Acharya, R.R. 2017. Bioefficacy of Cyantraniliprole 10\% OD W/V (HGW86 10 OD) against Pests of Potato. Int.J.Curr.Microbiol.App.Sci. 6(7): 309-317.

doi: https://doi.org/10.20546/ijcmas.2017.607.036 